\section{Gene drive}

Initially coined to describe the process of stimulating biased inheritance of particular genes to alter entire populations, the term is now increasingly used to describe the actual synthetic genetic element designed to increase in frequency over time in a population. In this Review, this term is inclusive of all types of gene drive systems discussed and is used interchangeably with the term 'selfish genetic element'.
Department of Entomology. University of California, Riverside, Center for Disease Vector Research, Institute for Integrative Genome Biology, University of California, Riverside, California 92521, USA.

Correspondence to O.S.A omar.akbari@ucr.edu

* These authors contributed equally to this work.

doi: 10.1038/nrg.2015.34

Published online 15 Feb 2016

\title{
Cheating evolution: engineering gene drives to manipulate the fate of wild populations
}

\section{Jackson Champer*, Anna Buchman* and Omar S. Akbari}

Abstract | Engineered gene drives - the process of stimulating the biased inheritance of specific genes - have the potential to enable the spread of desirable genes throughout wild populations or to suppress harmful species, and may be particularly useful for the control of vector-borne diseases such as malaria. Although several types of selfish genetic elements exist in nature, few have been successfully engineered in the laboratory thus far. With the discovery of RNA-guided CRISPR-Cas9 (clustered regularly interspaced short palindromic repeats-CRISPR-associated 9) nucleases, which can be utilized to create, streamline and improve synthetic gene drives, this is rapidly changing. Here, we discuss the different types of engineered gene drives and their potential applications, as well as current policies regarding the safety and regulation of gene drives for the manipulation of wild populations.

The possibility of addressing major biological problems related to public and environmental health by releasing transgenic organisms into the wild was first proposed decades $\mathrm{ago}^{1-3}$ and continues to be of substantial interest ${ }^{4,5}$. However, the field of molecular biology has struggled to live up to the great potential promised by these theoretical considerations. For example, although genetic approaches have been proposed to combat diseases transmitted by mosquitos ${ }^{1}$, such diseases remain a global burden and currently infect hundreds of millions of people per year, often with devastating consequences ${ }^{6,7}$; malaria alone is responsible for nearly half a million deaths annually, mostly among children ${ }^{8}$.

Gene drive systems (or gene drives) possess the potential to provide revolutionary solutions to key public health and environmental issues ${ }^{1,2}$. Most proposed engineered gene drives are based on naturally existing 'selfish' genetic elements that function by increasing in frequency with each generation even without conferring a fitness advantage upon their host, thus forcing non-Mendelian inheritance patterns ${ }^{9-15}$. They may enable us to potentially overcome the evolutionary disadvantages of certain desirable traits and spread these traits throughout wild populations, or to suppress populations of target species altogether ${ }^{9-13}$. This, in turn, may enable the development of novel strategies (BOX 1), for example, to reduce or eliminate insect-borne diseases, remove invasive foreign species, and even reverse the development of resistance to insecticides and herbicides, in an economically viable and environmentally friendly manner. Gene drives could also be used to spread advantageous traits through populations far more quickly and thoroughly than natural selection - for example, to provide aid for endangered species by spreading pathogen-resistant payload genes through exposed populations.

Despite the wide-ranging applicability and vast importance of gene drives, the past decades have seen only modest progress in their development. Gene drives that are capable of functioning in wild populations have been created in merely a handful of organisms, including yeast ${ }^{16}$, the fruitfly Drosophila melanogaster ${ }^{17-20}$, and two species of mosquitoes: Anopheles stephensi ${ }^{21}$ and Anopheles gambiae ${ }^{22}$. This is, in part, due to the difficulty of engineering the genomes of even model organisms. However, recent advancements in molecular and synthetic biology have provided broadly applicable tools that are capable of engineering the genomes of diverse species, which show great potential for creating a variety of engineered gene drives in diverse organisms. The most promising of these tools is the RNA-guided CRISPR-Cas9 (clustered regularly interspaced short palindromic repeats-CRISPR-associated 9) endonuclease system ${ }^{23}$, which can be used in conjunction with small guide RNAs (gRNAs) to cleave a specific sequence. Remarkably, this system can be designed to universally target virtually any genomic sequence, and has recently been adapted to modify the genomes of yeast ${ }^{16}$, plants ${ }^{24}$, worms ${ }^{25}$, fruitflies ${ }^{17,26}$, mosquitoes $^{21,22,27,28}$, zebrafish ${ }^{29}$, mice $^{30}$, monkeys ${ }^{31}$ and human 
Payload genes

Genes that can be linked to a gene drive to spread a

desirable trait throughout a population.

\section{CRISPR-Cas9}

(Clustered regularly

interspaced short palindromic repeats-CRISPR-associated 9)

A gene editing technology originating in bacteria that consists of an endonuclease (Cas9) and a guide RNA that can target and modify user-defined DNA and RNA sequences with great accuracy.

Resistance alleles

Alleles that are resistant to a drive system, preventing it from spreading. They can originate from mutations or errors in replication or DNA repair resulting from the gene drive, or they can exist in a population before release of the gene drive.

RNA-guided endonucleases Nucleases such as

CRISPR-associated 9 (Cas9) or $\mathrm{Cpf} 1$, that are directed by guide RNAs to target and cleave specific nucleotide sequences.

Cpf1

A programmable RNA-guided endonuclease from bacteria that cleaves DNA, generating staggered double-stranded breaks

Homing

The process by which an endonuclease cleaves a specific DNA target sequence and copies itself, or 'homes', into this target sequence. Homing utilizes the cell's homology-directed repair (HDR) machinery, which relies on sequences that flank the endonuclease and that are homologous to either side of the target sequence. The ultimate result of 'homing' is to generate an exact copy of the endonuclease in the target sequence.

Removability

The ability to completely remove a gene drive system from a population via the release of wild-type organisms.

Reversibility

The ability to replace an existing gene drive system with another system cells ${ }^{32}$, among others. The Cas9 endonuclease system has several important characteristics that make it especially attractive for use in engineering gene drives. RNAguided Cas 9 can be utilized to induce cleavage of DNA followed by homology-directed repair (HDR) from a co-delivered DNA cassette ${ }^{17}$, thus enabling precise genomic manipulations. Furthermore, in addition to cleaving DNA, Cas9 has recently been shown to be capable of cleaving mRNA ${ }^{33,34}$, thus broadening its potential use for developing gene drives. Moreover, gRNAs can be multiplexed for simultaneous Cas9-mediated targeting of multiple sequences ${ }^{35,36}$, permitting increased cleavage rates and reduced evolution of resistance alleles. Also, available bioinformatics tools can aid in minimizing off-target cleavage and improving species specificity $^{37}$. Other RNA-guided endonucleases, such as the recently described Cpf1 (REF. 38), may also prove to be as adaptable as the Cas9 system.

In this Review, we explore how these contemporary tools can contribute to the creation of gene drives inspired from systems that naturally exist in the wild. We provide an overview of the background of several types of gene drives and the current progress and future considerations concerning their development, along with novel architectures enabled by RNA-guided endonucleases that may facilitate the advancement of these systems. Finally, we consider the safety policies needed to address the possible dangers of gene drives and highlight potential future applications of these systems.

\section{Principal characteristics of gene drives}

In sexual reproduction, each of the two alleles of any gene is transmitted to $50 \%$ of offspring. Gene drives are genetic elements that circumvent this rule, significantly increasing the probability that the offspring will inherit the allele containing a gene drive element rather than a wild-type allele. Because of this, a gene drive can spread through a population even if it carries a fitness cost to the organism, as individuals with a gene drive element will produce more offspring with the gene drive allele than without it. Although there is no single molecular mechanism underlying all gene drives, they typically induce biased inheritance patterns via one of two methods. The first strategy involves copying themselves onto the opposite chromosome (that is, homing), resulting in most or all offspring inheriting the gene drive allele. The second method involves reducing the viability of gametes that inherit the wild-type allele, thus giving the wildtype allele a fitness disadvantage compared to the gene drive allele.

Gene drives can be characterized by a number of different attributes, including rate of spread, species specificity, fitness cost, susceptibility to resistance, removability and reversibility (TABLE 1); these attributes are of varying importance in different biological and regulatory scen$\operatorname{arios}^{15}$. Engineered gene drives can be classified as either 'modification drive' types, which are designed to spread through a population carrying desirable traits (for example, payload genes), or as 'suppression drive' types, which

\section{Box 1 | Applications of gene drives}

Effective gene drives may enable us to control invasive species, re-sensitize organisms that have developed resistance to insecticides and herbicides, and reduce or eliminate many types of vector-borne diseases, all at a low $\operatorname{cost}^{49}$. This latter objective, particularly as it applies to malaria, has been the main focus of research into gene drives.

Considerable progress has been made in assessing payload genes for the reduction of malaria transmission by mosquitoes ${ }^{140,141}$, some of the most promising of which are an AKT transgene expressed in the midgut ${ }^{142}$ and a single-chain antibody expressed in the salivary gland ${ }^{143}$. Dengue-refractory mosquitoes have also been engineered ${ }^{144,145}$, but these only confer resistance to dengue-2, which is just one of five known serotypes. It may be possible to develop mosquitoes that are refractory to all dengue serotypes by using an RNA-guided endonuclease with specific guide RNAs targeting the RNA genome of each serotype, but this remains to be demonstrated. In addition to malaria and dengue, payload genes that confer resistance to Zika virus could also foreseeably be developed using either an RNA interference (RNAi) type of approach, or by expressing an RNA-guided endonuclease combined with guide RNAs designed to target the single-stranded RNA genome of Zika virus. Although not yet developed, other payload genes of great practical importance may immunize threatened or agriculturally important organisms against pathogens, such as genes that make the citrus psyllid - the most important pest of citrus worldwide ${ }^{146}$ - unable to transmit huanglongbing (HLB) disease, or genes that render amphibians immune to the killer Chytrid fungus, which is responsible for the decline of amphibian species all over the world ${ }^{147}$.

In addition to carrying payload effectors, homing-based drives or meiotic drives may also be utilized for population suppression of both disease vectors and economically or ecologically damaging invasive species, particularly those with short generation times. Additionally, the versatility of RNA-guided endonucleases may allow for other suppression approaches, such as the reversal of resistance to pesticides or herbicides by specifically targeting resistance alleles and replacing them with sensitive ones - a process that could be repeated if resistance is reacquired. Another possibility would be to insert a payload gene that activates during insect diapause, causing lethality. Gene drive systems could also introduce a sensitizing gene that renders a population vulnerable to an otherwise harmless molecule, allowing a species to be specifically targeted with minimal ecological disruption.

For gene drives, the evolutionary stability of the payload is a major concern, as any inactivating mutations may lead to the spread of a non-functional system ${ }^{39}$. The effects of such mutations can be minimized by using compact payloads, multiple unique payloads, and multiple recoded copies of each payload gene. However, care must be taken to minimize the fitness cost imposed on the organism by payload genes. In the case of an inactivated payload, release of second-generation gene drives can replace these with functional copies. Overall, in addition to the disease-refractory genes already characterized, there is a plethora of yet to be developed potential payload genes that may be of great utility to human health, agriculture and the environment. The engineering of functional gene drives in important species is likely to further spur their development. 
Table 1 | Comparison of the various types of gene drive systems

\begin{tabular}{|c|c|c|c|c|c|c|}
\hline & $\begin{array}{l}\text { Homing-based } \\
\text { drive }\end{array}$ & X-Shredder & Medea & $\begin{array}{l}\text { Toxin-antidote } \\
\text { underdominance }\end{array}$ & $\begin{array}{l}\text { Chromosomal } \\
\text { rearrangement }\end{array}$ & Wolbachia \\
\hline Type & Either & Suppression & Replacement & Replacement & Replacement* & Replacement $^{\ddagger}$ \\
\hline Rate of spread & Fast & Moderate & Moderate & Slow & Slow & Moderate \\
\hline Locally confined? & No & No & $\begin{array}{l}\text { No, if low } \\
\text { fitness cost }^{\S}\end{array}$ & Yes & Yes & $\begin{array}{l}\text { No, if low fitness } \\
\operatorname{cost}^{\S}\end{array}$ \\
\hline $\begin{array}{l}\text { Resistance allele } \\
\text { generation rate }\end{array}$ & High & Low & Low & Moderate & Very Low & Unknown \\
\hline $\begin{array}{l}\text { Removable with } \\
\text { wild type? }\end{array}$ & Noll & Noll & $\begin{array}{l}\text { No, if low } \\
\text { fitness cost }^{\S}\end{array}$ & Yes & Yes & $\begin{array}{l}\text { No, if low fitness } \\
\operatorname{cost}^{\S}\end{array}$ \\
\hline Status & $\begin{array}{l}\text { Drosophila }{ }^{17} \text {, } \\
\text { Saccharomyces }^{16}, \\
\text { Anopheles stephensi }^{21} \text {, } \\
\text { Anopheles gambiae }^{22}\end{array}$ & $\begin{array}{l}\text { Incomplete in } \\
\text { Anopheles gambiae }{ }^{76}\end{array}$ & Drosophila ${ }^{19,20}$ & Drosophila ${ }^{18,96}$ & $\begin{array}{l}\text { Natural } \\
\text { examples }^{89,94}\end{array}$ & Field tests ${ }^{111,112}$ \\
\hline
\end{tabular}

The characteristics listed here are variable and depend on a range of factors (for example, ecology of the target species, population distribution, movement patterns, fitness costs, payload characteristics, and so on); therefore, only ideal-case scenarios are compared to emphasize intrinsic differences of the various types of drives. ${ }^{\star}$ Chromosomal rearrangement can be used for short-term population suppression. ${ }^{\ddagger}$ It is possible that male-killing strains of Wolbachia may be usable for population suppression. ${ }^{\S}$ High fitness costs may make these systems locally confined and removable with the release of large numbers of wild-type organisms. "Suppression types that proceed to fixation and eliminate a population will remove the gene drive system, allowing replacement with wild-type organisms.

Modification drive A gene drive designed to spread genomic changes and/ or genetic payloads throughout a population, thereby modifying the population.

Suppression drive A gene drive designed to reduce or eliminate the population of its target organism

Homing endonuclease genes

(HEGs). Naturally occurring types of gene drive that are composed of an endonuclease encoded either as a

freestanding gene within introns, as a fusion with host proteins, or as a self-splicing intein, with the ability to home into the opposite chromosome resulting in more than half of offspring inheriting the HEG. have the effect of reducing the population of a target species (FIGS 1,2). They can be further subdivided based on how they spread through a population.

Fast-spreading gene drives, which are typically considered invasive, have low initial release requirements, whereas gene drives that spread more slowly and are confined to a local breeding population require high release thresholds. Generally, the specificity of gene drives for a target species is highly desirable to ensure containment, as is a low fitness cost to the carrying organism. It may seem counterintuitive to have a low fitness cost when using gene drives to suppress a population; however, the drive system itself should be able to efficiently suppress populations, and an intrinsic fitness cost of such a system would slow down its rate of spread, thus reducing its long-term suppressive ability. Another characteristic that varies between different types of gene drives is the likelihood that resistance will develop in the target species in response to the drive, which can take the form of natural resistance alleles or be induced by the gene drive itself ${ }^{39}$. In most cases, low resistance is desirable unless resistance is part of a scheme to confine the gene drive to a smaller geographical area. Another important feature is the evolutionary stability of a gene drive, which refers to the predisposition of the gene drive and its cargo to persist and remain active in a population in the long term ${ }^{39}$.

Given their potential to alter entire ecosystems ${ }^{40,41}$, it may be advantageous for gene drive systems to be either completely removable from a population by the release of wild-type organisms (in the case of high-threshold gene drives) or convertible to a neutral configuration by the construction of a secondgeneration gene drive, which is frequently denoted as a 'reversal' gene drive. Of note, despite their name, reversal gene drives do not restore the original modification to the wild type; rather, they induce further changes that may undo a phenotypic alteration caused by the initial gene drive. The properties discussed above all need to be considered when evaluating the type of gene drive that is best suited for a particular application and assessing context-dependent risks.

\section{Homing-based drives}

First proposed for use in manipulating populations by Burt in 2003 (REF. 4), homing endonuclease genes (HEGs) possess high rates of drive and can be exploited for both population suppression and modification. HEGs have the remarkable intrinsic ability to 'cheat' during meiosis by converting their corresponding allele on the opposite chromosome into an exact copy of themselves ${ }^{42}$ (FIG. 1a). They achieve this feat by encoding a sequence-specific endonuclease that severs and disrupts their competing chromosomal allele, forcing the cell to rapidly repair this DNA break. Upon breakage, the cell has several repair options: it can stitch the damaged ends together using

Figure 1 | Mechanisms of homing drives. a | A homing endonuclease gene (HEG) works by encoding an endonuclease, which cleaves at a target site on the homologous chromosome opposite the HEG. Homologydirected repair (HDR) results in the HEG being copied to the homologous chromosome. $\mathbf{b} \mid$ A homing element may be generated using an RNA-guided CRISPR (clustered regularly interspaced short palindromic repeats) endonuclease together with one or more small guide RNAs (gRNAs). Resistance alleles can be minimized by targeting the homing-based RNA-guided drive to a conserved critical gene at multiple locations using several gRNAs. The gene would only be reformed to functionality if HDR takes place, precluding successful repair and induction of resistance alleles by non-homologous end joining (NHEJ). c $\mid$ A homingbased RNA-guided drive may be removed from a population by designing a reversal drive encoding a gRNA that targets the previous generation drive. $\mathbf{d} \mid$ A homing drive may be utilized to suppress a population by homing into a critical gene, the disruption of which induces recessive sterility (in this example, female infertility) or lethality. 
a

HEG

Homologous chromosome

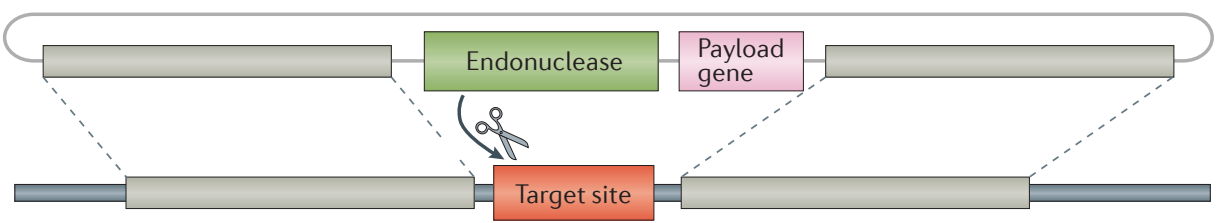

$\downarrow$ Homology-directed repair

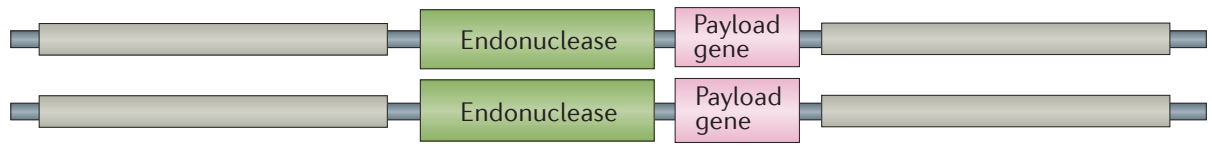

b

RNA-guided

drive

Homologous chromosome
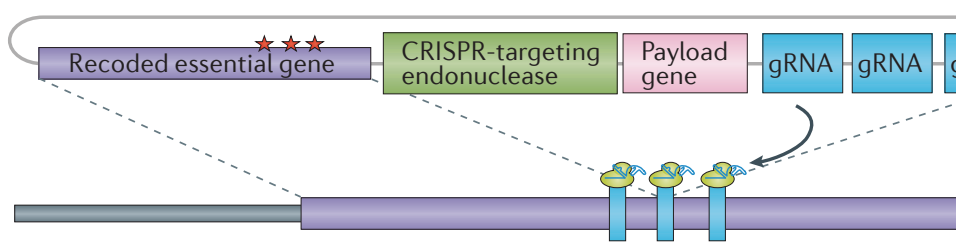

Target sites

$\downarrow$ Homology-directed repair

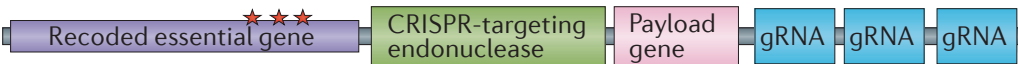

Recoded essential gene

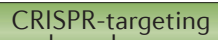

Payload

gene

gRNA gRNA $=$ gRNA

Reversal drive

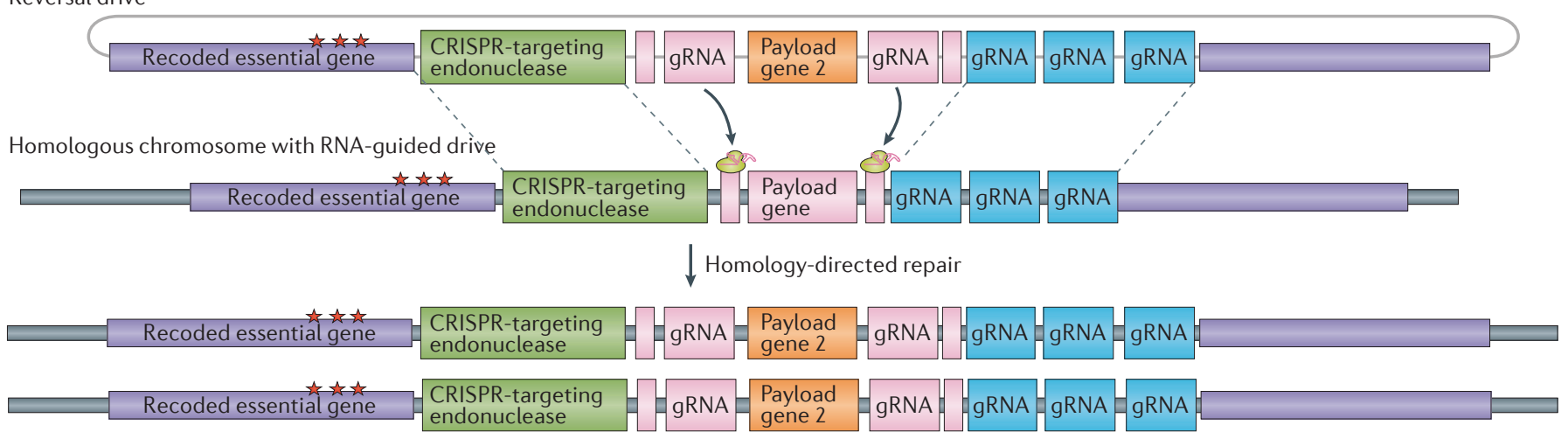

d

Suppression

drive

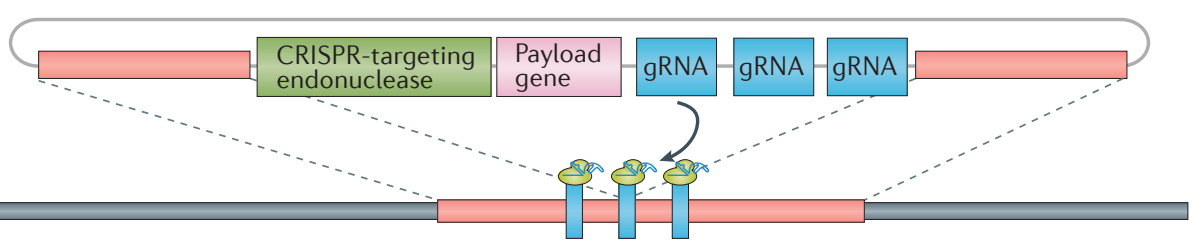

Recessive viability or fertility gene

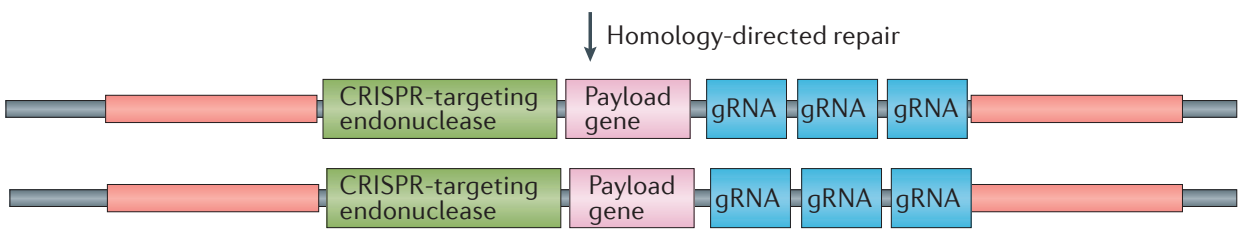

Recessive viability/fertility gene disrupted by drive 

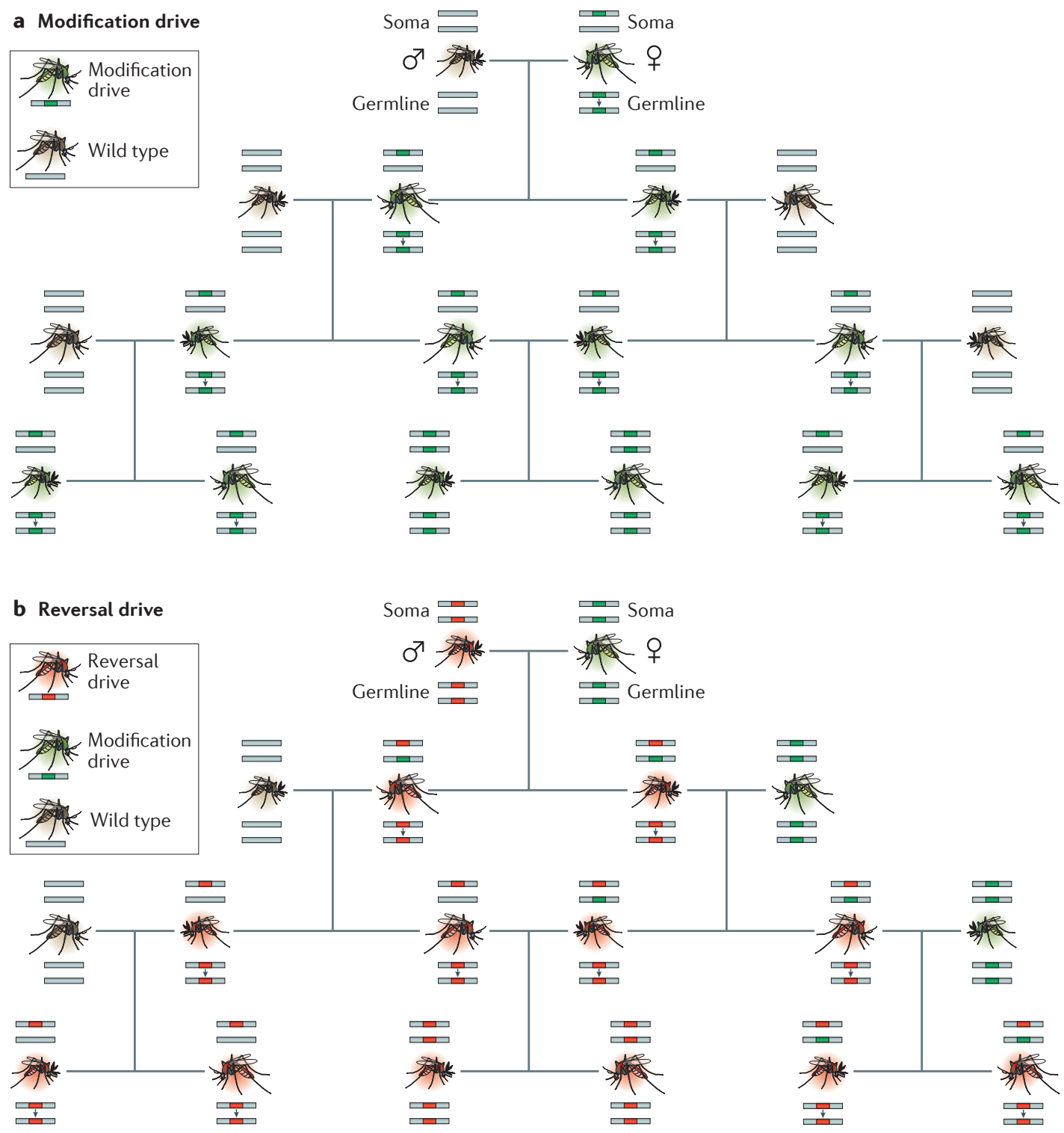

c Suppression drive

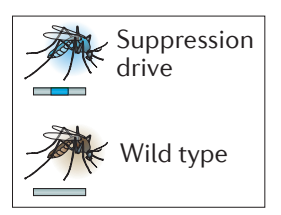

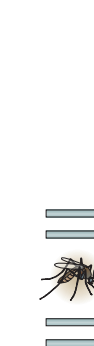


Homing efficiency

The rate at which a homing-based drive gene becomes successfully copied onto the opposite

chromosome via homology-directed repair

RNA-guided drive Any engineered drive system that utilizes an RNA-guided endonuclease to bias its inheritance and increase in frequency in a population. either the non-homologous end joining (NHEJ) or microhomology-mediated end joining (MMEJ) pathways, both of which can potentially form HEG-resistant alleles, or it can use the HEG as a template for HDR, resulting in the HEG copying itself (that is, homing) into its competing allele ${ }^{42}$. If HDR occurs in the germ line or early embryo, the proportion of offspring that receive the HEG will be higher than 50\% (the proportion expected from normal Mendelian transmission), allowing for rapid invasion of the HEG into a target population, along with any payload genes that are designed to 'piggyback' on the HEG $^{43-49}$ (FICS 1a,2a). Besides spreading a genetic payload, a HEG may also be utilized for population suppression, and possibly eradication, by homing into a target gene, the disruption of which leads to recessive lethality or sterility $^{4,44}$. In such a suppression approach, homing must be confined to the germ line during gamete formation, leading to sterility or non-viability only in homozygotes that receive the HEG allele from both parents. Consequently, the HEG can rapidly spread, and once a large fraction of the population is heterozygous it will cause a population crash as heterozygote pairings will produce sterile or non-viable offspring. HEGs may also be designed to manipulate populations by targeting other suitable genes, such as genes to reduce lifespan, to bias sex ratios, to impede host seeking, to block pathogen development, or to block the ability of the modified organism to act as a vector for pathogens.

Several proof-of-principle studies have successfully demonstrated the feasibility of using HEGs as gene drives ${ }^{50-53}$. However, these studies were limited by utilizing HEGs that targeted artificially inserted nuclease recognition sites. Re-engineering naturally occurring HEGs to target non-innate recognition sequences is possible ${ }^{54}$, but it requires complex protein engineering and has proven to be exceedingly difficult. Although progress has been made in using alternative nucleases with recognition sequences that are straightforward to engineer, such as transcription activator-like effector nucleases (TALENs) and zinc-finger nucleases (ZFNs), the genes encoding them are exceptionally large and repetitive in nature, making them less evolutionary stable and thus less efficient for use in gene drives ${ }^{55}$. The recent discovery and characterization of RNA-guided endonucleases such as Cas9 (REF. 56) and Cpf1 (REF. 38) may serve to overcome such limitations ${ }^{49}$. In fact, Gantz and Bier ${ }^{17}$ recently successfully engineered a homing RNA-guided system that functions as a potent gene drive in the fruitfly with a remarkable $96 \%$ homing efficiency. Since then, homing RNA-guided drive systems ${ }^{49}$ based on Cas9 with similarly

4 Figure 2 | Spread of homing drives. a $\mid$ A homing drive results in most or all progeny of heterozygotes receiving the homing element, which allows the drive to spread rapidly throughout the population. $\mathbf{b} \mid$ A second-generation reversal drive can overwrite a first-generation homing drive, replacing its payload gene. Progeny of heterozygotes with this drive will all inherit the second-generation drive. This homing drive may be configured to home into wild-type alleles as well, immunizing the population against the first-generation homing drive. $\mathbf{c} \mid$ A suppression drive targeting a recessive gene required for viability or fertility will spread rapidly from heterozygotes with the drive, but would create an increasing number of sterile or unviable homozygotes, eventually resulting in a population crash. high homing frequencies have been developed in yeast ${ }^{16}$ and mosquitoes ${ }^{21,22}$. For example, a study in A. stephensi demonstrated the ability of a homing modification drive system to spread a large payload containing an antimalarial single-chain antibody; however, the homing element did not function properly in the progeny of females, making the system unstable ${ }^{21}$. In another study in A. gambiae, researchers created a suppression drive targeting female fertility genes ${ }^{22}$. This drive was successfully transmitted to offspring, although its population suppression capability was limited because heterozygous disruption of the target genes greatly reduced female fertility. Together, these innovative efforts provide the first examples of flexible and highly efficient RNA-guided homing drives.

Future studies should focus on adapting these homing RNA-guided drive approaches to other organisms and attempt to improve the capabilities of RNA-guided endonucleases, which will be useful for the development of both homing-based drives and other forms of gene drives. Specifically, efforts to maximize HDR and to minimize the NHEJ and particularly more error-prone MMEJ pathways could increase the rate of spread and reduce the creation of resistance alleles, which are likely to arise when end joining takes place. Methods to achieve this may include careful selection of target sequences to reduce the frequency of MMEJ ${ }^{57}$, expression of the endonuclease only in the germ line during meiosis to increase the rate of $\mathrm{HDR}^{58,59}$, blockage of ligase $\mathrm{IV}^{60}$ and other components required for NHEJ, and multiplexing of gRNAs targeting a conserved sequence in an essential gene so that it is only re-formed during HDR (FIG. 1b), which would render NHEJ products non-viable and HDR products functional.

Unfortunately, these methods will not completely eliminate the formation of resistance alleles, as HDR is known to have an error rate itself ${ }^{148}$, which may limit the propagation of a homing drive ${ }^{39}$. If the gene drive is not copied perfectly, it could be inactivated, generating a drive resistance allele. Such resistance alleles may have increased fitness compared to the original drive - for example, if they express fewer active components (such as the payload gene and nuclease) - and therefore spread in the population, outcompeting the original drive. Resistance alleles to homing-based drives could also arise if the gRNA target sites are mutated in-frame by NHEJ or MMEJ, and such mutated sequences would be strongly selected for in the presence of a suppression drive. Indeed, in the recently described Cas9-based drive systems, resistance alleles were detected within a few generations ${ }^{16,17,21,22}$, and some form of resistance was also reported in all previous studies utilizing homing-based drives ${ }^{50,51,53-55}$, highlighting the fundamental instability of engineered homing-based systems. Strategies to improve the evolutionary robustness of synthetic homing-based drives should therefore be further investigated, along with the long-term stability of payload genes. For example, steps should be taken to minimize off-target cleavage, which may reduce the fitness of the organism or lead to the generation and inadvertent spread of undesirable mutations. This can be undertaken using both predictive software ${ }^{37,61,62}$ and endonucleases with improved specificity ${ }^{63,64}$. 


\section{X-shredder \\ In an X-Y heterogametic species, an X-Shredder is a type of gene drive that cleaves the $\mathrm{X}$ chromosome at multiple places during meiosis in males, thus destroying it. Because of this, most or all of the viable sperm will contain Y chromosomes, resulting in biased sex ratios in favour of males and, over time, suppression of the population owing to lack of females. \\ Sterile insect technique A method for temporarily suppressing target populations, whereby overwhelming numbers of mass-produced sterile insects are released to mate with wild-type insects.}

\section{Heterogametic}

A species containing different sex chromosomes between males and females. Humans are an example of a heterogametic species; Y chromosomes are found only in males.
Finally, it should also be noted that, once homingbased drives are released, complete reversion of a population to a wild-type genotype is not possible. However, modification of fixed systems is feasible using functional resistance alleles, trans-acting reversal elements, second-generation drives comprising gRNAs designed to target and replace only the original drive system, or even fully functional second-generation drives based on RNA-guided endonucleases that are capable of spreading through both wild-type populations and those modified by the first-generation drive ${ }^{48,49}$ (FICS 1 c,2b). Overall, although various crucial questions regarding their utility remain, homing RNA-guided drives seem promising for both population modification and suppression (FICS 1d,2c), and efforts to try to improve their capabilities, robustness and stability on an evolutionary timescale should be of high priority.

\section{Sex-linked meiotic drives}

Meiotic drive occurs when transmission of certain alleles is biased during meiosis, resulting in increased frequencies of those alleles in the gametes, and hence in the offspring ${ }^{65,66}$. Many types of meiotic drive systems are found in nature, including sex-linked meiotic drive elements that function by skewing gender ratios ${ }^{5,66,67}$. These sex-linked meiotic drive systems typically operate by preventing the maturation of gametes that lack the meiotic driver and have been identified in several species ${ }^{68}$. The use of such systems was first suggested for population suppression over 50 years ago ${ }^{2,69,70}$, and naturally occurring forms of Y-chromosome-linked drive (Y-drive) have been identified and shown to suppress insect populations by increasing the proportion of males ${ }^{67,71}$. Although the molecular mechanisms of natural Y-drives are unknown, cytology indicates that in these systems the $\mathrm{X}$-chromosome is broken during male mei$\mathrm{osis}^{72}$. To mimic the effects of this natural Y-drive, it has been proposed that an engineered meiotic drive-based system could be developed based around an endonuclease that destroys the $\mathrm{X}$ chromosome in an X-Y heterogametic species $^{4,44}$. Such an X-chromosome shredder (X-shredder) may consist of an endonuclease that functions by targeting and cutting several locations on the $\mathrm{X}$ chromosome during spermatogenesis, thus ensuring that only sperm with Y chromosomes are viable (FIG. 3a). If located on an autosomal chromosome, an X-shredder could be used to suppress populations, but it will be self-limiting and will necessitate substantial inundation and continuous release, similar to that required with the sterile insect technique ${ }^{12,73}$. Conversely, if the X-shredder element is localized to the $\mathrm{Y}$ chromosome, all of a male's offspring will inherit it, allowing it to quickly invade and suppress a population owing to an eventual reduction in the number of females.

Windbichler and colleagues ${ }^{51,74,75}$ engineered such an X-shredder in A. gambiae by expressing the I-PpoI endonuclease during spermatogenesis to target ribosomal repeats on the $\mathrm{X}$-chromosome, albeit from an autosomal location. They were able to achieve highly efficient paternal shredding of the X-chromosome with a modified version of I-PpoI that produced profoundly distorted sex ratios $(>90 \% \text { male })^{76}$. Efforts to localize this system to characterized Y-chromosome docking lines ${ }^{77}$ in A. gambiae are underway (A. Crisanti, personal communication). However, although the use of I-PpoI as an $\mathrm{X}$-shredder in A. gambiae is promising, it is unlikely to be portable across species, as it will only function in the few organisms that have an X-chromosome with repeated I-PpoI target sequences. Therefore, the development of other endonuclease technologies that can be easily transferred across species is of high priority. Given their highly flexible sequence targeting, RNA-guided endonucleases may be used to overcome this limitation to engineer gene drives that function as $\mathrm{X}$-shredders. These could be localized to the Y chromosome and express gRNAs that target highly conserved, species-specific regions of an X chromosome (FIG. 3b).

In general, an RNA-guided meiotic drive system could be designed to function in most heterogametic species and may allow for population suppression of many target organisms. However, potential resistance alleles, generated through NHEJ, are of high concern, as natural selection tends to favour equal sex ratios ${ }^{2}$, and any resistance allele - even if arising in a single individual - will probably spread rapidly in the presence of the drive. Therefore, it will be important to target multiple essential conserved genes on the X chromosome to minimize the evolution of resistance arising from target site mutations. Moreover, similarly to the homing-based gene drives discussed above, complete reversion of X-shredders to wild-type genotypes is not possible, although they can be inactivated by a suppressor with the use of a second-generation drive 'reversal' element (FIG. 3c).

\section{Medea}

The maternal effect dominant embryonic arrest (Medea) system was first discovered in the flour beetle ${ }^{78}$. The molecular underpinnings of the natural Medea system remain unknown, but multiple versions of the Medea inheritance pattern have been reverse engineered and shown to act as robust gene drives in D. melanogaster ${ }^{19,20}$. These engineered Medea systems behave as modification drives by utilizing an RNA interference (RNAi)-based toxin-antidote combination. The toxin consists of a microRNA (miRNA) that is expressed during oogenesis in Medea-bearing females, disrupting an embryonic essential gene in all embryos, regardless of whether those embryos have inherited a Medea or wild-type allele from the mother (FIG. 4a). A tightly linked antidote consisting of a recoded version of the target gene that is immune to the effect of the miRNA - is expressed at the zygotic stage early in embryogenesis, only in those embryos that inherit the Medea element. This creative combination of maternally expressed toxin and a zygotically expressed antidote results in the survival of $50 \%$ of the embryos originating from a Medea-bearing heterozygote female, as those that fail to inherit the Medea element perish. Moreover, if the female has mated with a Medea-bearing heterozygous male, the antidote from the male will also take effect in the embryo, resulting in $75 \%$ of the embryos surviving (FIG. 4 a). Consequently, Medea possesses a frequency-dependent 
a

Y chromosome with X-shredder

X chromosome

Cleaved

X chromosome

b

Y chromosome with RNA-guided X-shredder

X chromosome

Cleaved

X chromosome

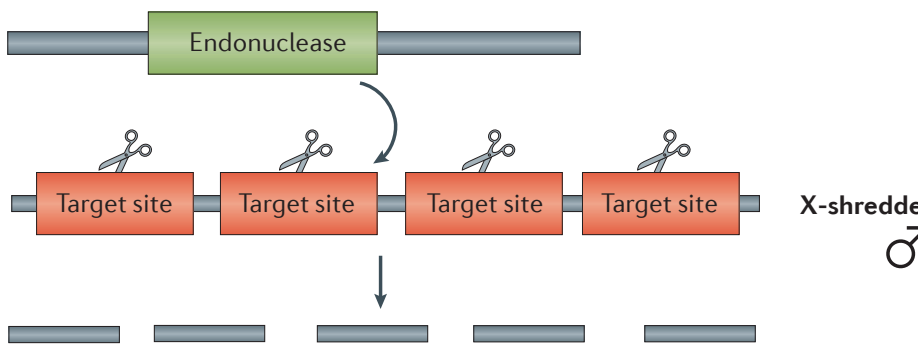

Wild type \{

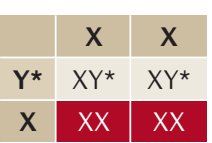

c

X chromosome with reversal element

Y chromosome with RNA-guided

X-shredder

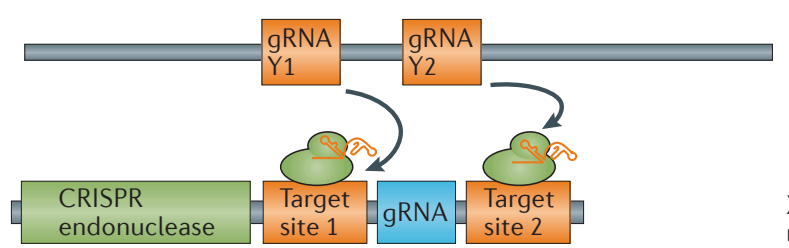

\begin{tabular}{|c|c|c|c|} 
& \multicolumn{3}{c}{ Wild type } \\
& & $\mathrm{X}$ & $\mathrm{X}$ \\
\hline $\begin{array}{l}\text { X-shredder/ } \\
\text { reversal }\end{array}$ & $\mathrm{Y}^{*}$ & $\mathrm{XY}^{*}$ & $\mathrm{XY*}$ \\
\hline $\mathrm{O}^{*}$ & $\mathrm{X}^{*}$ & $\mathrm{XX}^{*}$ & $\mathrm{XX^{* }}$
\end{tabular}

Y chromosome with disrupted RNA-guided X-shredder

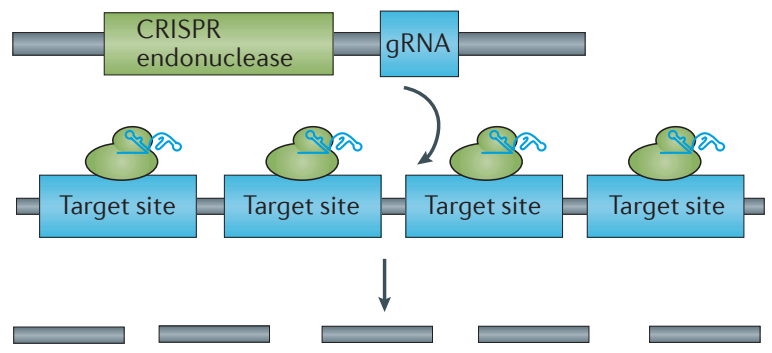

Figure 3 | Designs for Y-drive systems. a | An X-chromosome shredder (X-shredder) system works by expressing an endonuclease from the $Y$ chromosome, in an $X$ - $Y$ heterogametic species, that cleaves the $X$ chromosome at many locations. This destroys the $X$ chromosome, so all viable sperm have only $Y$ chromosomes, leading to all male offspring and, eventually, population suppression. A red background denotes lethality. $\mathbf{b} \mid$ An RNA-guided CRISPR (clustered regularly interspaced short palindromic repeats) endonuclease with one or more small guide RNAs (gRNAs) may be used as the X-shredder. c | An RNA-guided endonuclease X-shredder can be reversed using an X chromosome containing multiple gRNAs targeting the gRNAs of the original X-shredder. These X-chromosome-localized gRNAs would be activated before the gRNAs on the $Y$ chromosome, resulting in removal of the gRNAs on the $Y$ chromosome before the $X$ chromosome is shredded. This permanently inactivates the $X$-shredder, resulting in increased production of female offspring, which have a major fitness advantage compared to male offspring when $\mathrm{X}$-shredder alleles remain in the population.

fitness advantage compared to chromosomes lacking Medea, allowing it to rapidly drive a linked payload gene through a population ${ }^{79-83}$.

Attempts to replicate synthetic Medea systems in the yellow fever mosquito Aedes aegypti and other species have not yet met with success (O.S.A., unpublished observations). One major limiting factor that has impeded their development is the lack of a basic understanding of how to achieve effective RNAi-mediated silencing of key genes in the germ line of species other than D. melanogaster. One possible method to overcome this limitation may be to develop Medea systems using an RNA-guided nuclease as the toxin designed to maternally target the mRNA of a maternally deposited embryonic essential gene, preferably in multiple places to increase evolutionary stability by reducing the likelihood of generating resistance alleles (FIG. 4b). This would enable straightforward mRNA targeting and would not require engineering complex miRNA hairpins that rely on nucleolytic processing to generate mature miRNAs. Moreover, this approach would also allow for the replacement of a previous Medea gene drive via the production of a reversal drive (FIG. 4c). Notwithstanding this, although RNA-guided endonucleases may facilitate the generation of efficient toxins, specific control sequences such as maternal and zygotic promoters and embryonic essential target genes will need to be identified and functionally characterized in the target species in order to engineer a functional Medea gene 
a

Chromosome with Medea
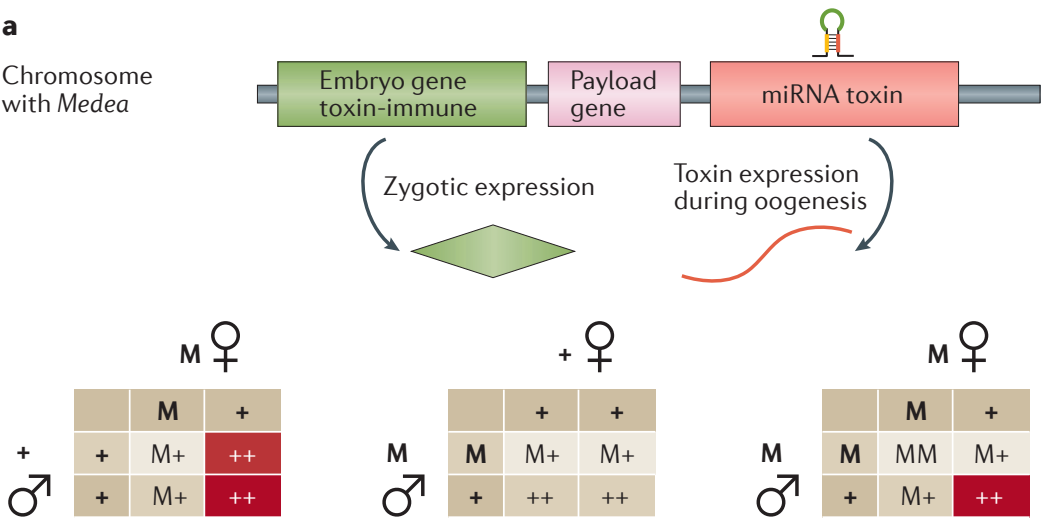

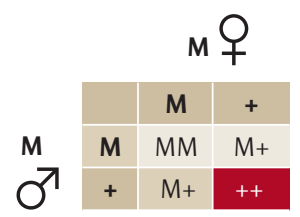

\section{b}

Chromosome with RNA-guided Medea
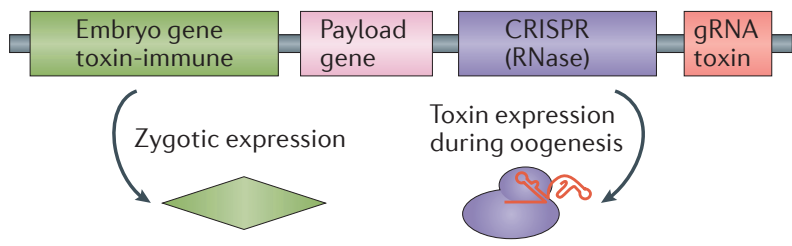

\section{C}

Reversal Medea (RM)
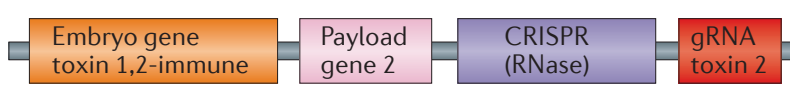

Original Medea (M)
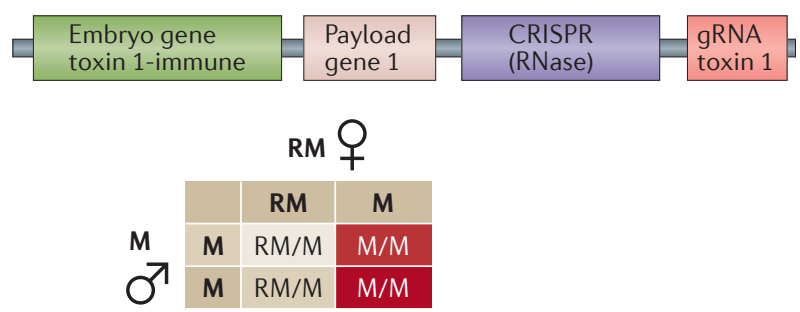

Figure 4 | Characteristics of Medea selfish genetic elements. a | The Medea system consists of a microRNA (miRNA) toxin expressed during meiosis that takes effect during embryonic development. The antidote consists of a protein expressed zygotically with an altered transcript sequence so as to be immune to the toxin. The system operates by killing offspring of a Medea-bearing female that fail to inherit Medea from either parent, resulting in frequency-dependent spread through a population. A red background denotes lethality. $\mathbf{b}$ | In lieu of an miRNA toxin, an RNA-guided CRISPR (clustered regularly interspaced short palindromic repeats) endonuclease may be used to cleave the mRNA of the target gene. $\mathbf{c}$ |A fixed Medea element can be reversed using a reversal Medea with a new toxin, a recoded antidote to both toxins (previous and new), and new payload. +, wild-type element; gRNA, guide RNA; M, original Medea element; $\mathrm{RM}$, reversal Medea element.

\section{Bistable switch}

A phenomenon in which a certain threshold frequency for

a gene drive system defines its eventual fate in a population.

If the frequency of individuals with the gene drive in a population is above that threshold, it will spread and eventually reach fixation. If it is below that threshold, it will be eliminated from the population. drive. Nevertheless, RNA-guided Medea systems hold promise as powerful systems that are capable of rapidly driving payload genes throughout wild populations.

\section{Underdominance gene drives}

Underdominance, also known as heterozygote inferiority, occurs when heterozygotes (or their progeny) have a lower fitness than parental homozygotes (FIG. 5a). Underdominant systems function as a bistable switch: even when two underdominant traits confer equal fitness in the homozygous state, the one with the lower initial frequency will be lost in a large interbreeding population, whereas the other will spread to fixation ${ }^{10,82-88}$. This occurs because individuals bearing the less common alleles are more likely to mate with the opposite type and produce unfit offspring, reducing the likelihood of the less common allele being passed on to future generations. Consequently, underdominant systems require a high introduction threshold to spread through a population, they are likely to be confined to a local area, and they can be removed by inundation with wild-type organisms (that is, the release of large numbers of wild-type organisms).

Balanced reciprocal chromosomal translocations are the classic example of underdominance, and were first proposed as a tool for population manipulation 75 years ago by Serebrovskii ${ }^{3}$ and later by Curtis ${ }^{1}$. Given that half of the progeny of translocation-bearing heterozygotes perish owing to an unbalanced gene set, translocationbearing individuals can be utilized both for population suppression $^{3}$ and for driving payload genes into populations ${ }^{1}$. Inversions and compound chromosomes are also types of chromosomal rearrangements that can be utilized to bring about population modification or suppression $^{89}$, and are regularly found in nature ${ }^{90}$.

The strategy of utilizing chromosomal translocations for population manipulation garnered a substantial amount of interest in the 1970s and $1980 \mathrm{~s}^{91}$, and some laboratory and field-cage experiments on mosquitoes were undertaken ${ }^{92-94}$. However, this area of research was ultimately abandoned as translocation-bearing individuals were generated via mutagens and typically had lower fitness than their wild-type counterparts ${ }^{91,95}$; moreover, there was no approach for linking a payload gene to the translocation breakpoint ${ }^{91}$. More recently, strategies to engineer underdominant gene drives using combinations of toxins and antidotes (FIG. 5b) have been proposed ${ }^{91}$ and implemented in the fruitfly D. melanogaster, both as a proof-of-principle system ${ }^{96}$, and as fully functional systems capable of invading wild populations ${ }^{18}$. However, to date, no engineered underdominant system capable of carrying a payload gene has been constructed in an organism of medical or agricultural relevance.

Although it might be possible to engineer toxinantidote underdominance gene drives in diverse species, developing small-RNA-based toxins could prove difficult. An alternative approach may be to use RNA-guided endonucleases targeting mRNA as a toxin element in an underdominance system (FIG. 5c). Moreover, RNA-guided endonucleases may offer a straightforward method to create sequence-specific chromosomal translocations at precise locations in a genome. For example, techniques for generating sequence-specific translocations via HDR following double-stranded DNA breaks ${ }^{97} \mathrm{can}$ be modified to make use of RNA-guided endonucleases (FIG. 5d). Such methods would be highly portable across organisms, and could generate species-specific underdominant gene drives that are profoundly evolutionarily stable, with no reliance on a large protein such as Cas9 for drive functionality and tight linkage of the payload gene to the translocation breakpoint, which is immune to meiotic recombination ${ }^{98}$. Although translocations may bear a fitness cost, the release threshold needed for the 

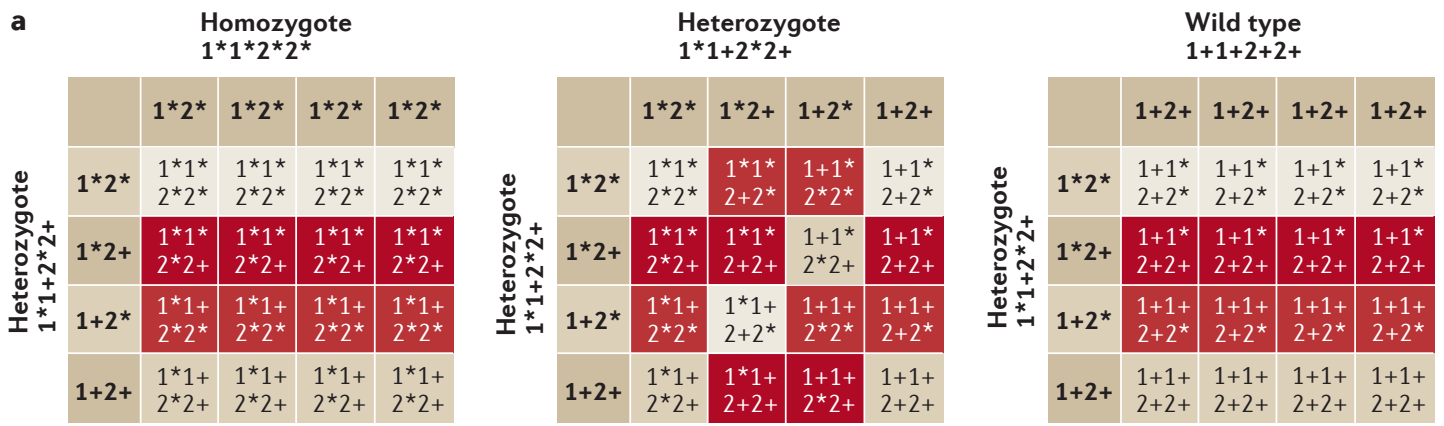

b
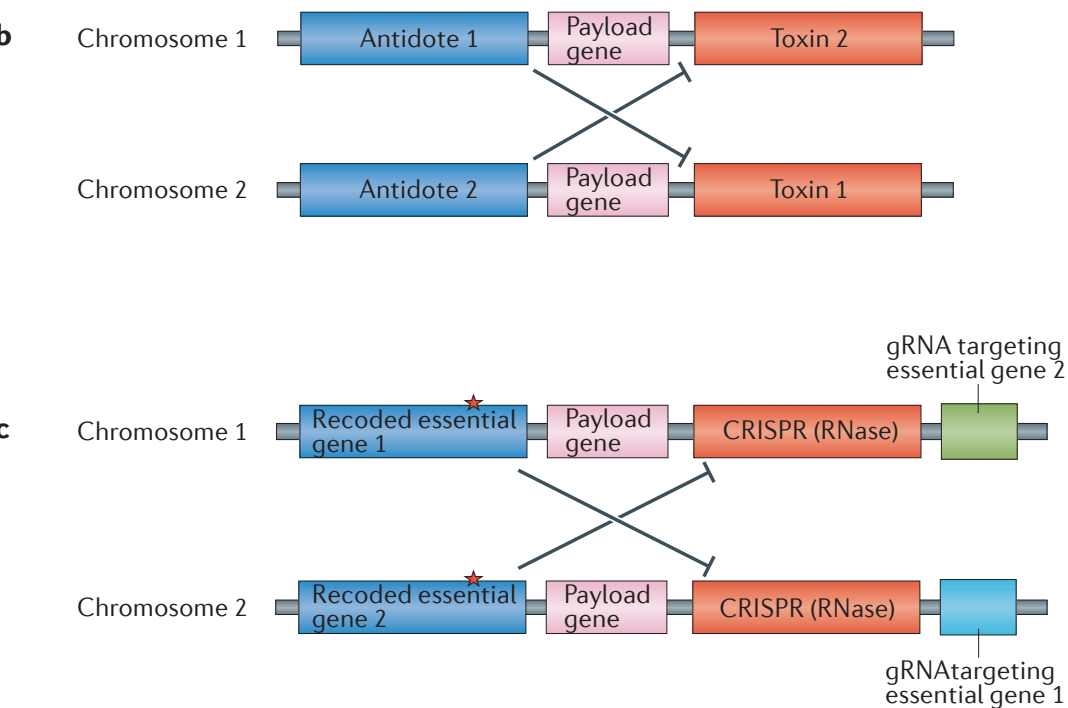

d

Chromosome 1

Chromosome 2
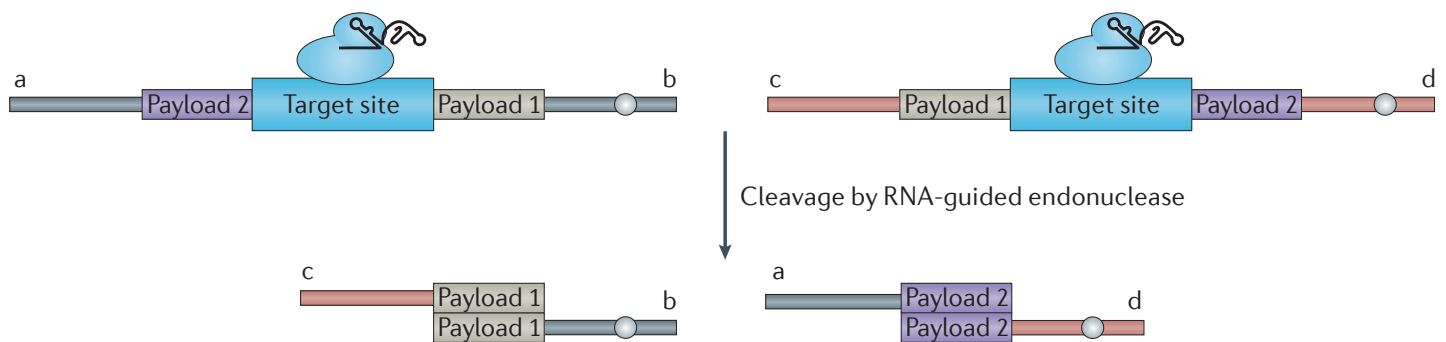

$\downarrow$

Cleavage by RNA-guided endonuclease

a
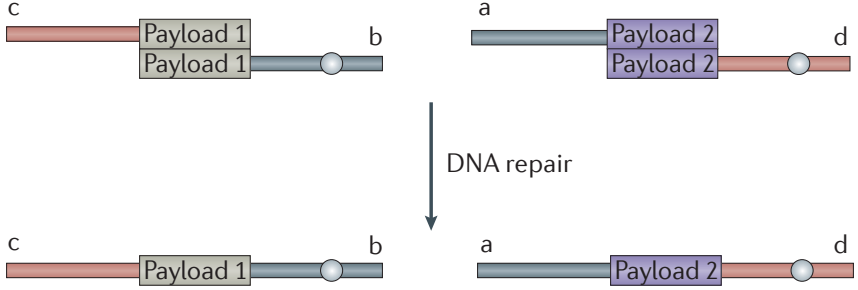

Figure 5 | Underdominance systems. a| Several variants of underdominance systems exist, including double toxinantidote systems and reciprocal chromosomal translocations. These systems function by reducing the fitness of heterozygotes to a greater extent than that of homozygotes. A red background denotes lethality. $\mathbf{b} \mid$ In two-locus toxinantidote systems, each element contains a toxin-antidote pair, requiring both to be present for an organism to be viable. c| These toxins can consist of RNA-guided CRISPR (clustered regularly interspaced short palindromic repeats) endonucleases that are designed to destroy an mRNA of an essential gene, whereas the antidote consists of resistant forms of the gene expressing mRNA that cannot be cleaved. $\mathbf{d}$ |RNA-guided CRISPR endonucleases may also be used to engineer chromosomal rearrangements to be used in underdominance systems. In part a, the numbers 1 and 2 refer to chromosomes, '+' denotes wild types, and * indicates transgenic organisms from reciprocal chromosomal translocations or the two-locus toxin-antidote system. In part d, lowercase letters a-d refer to chromosome arms. gRNA, guide RNA. 


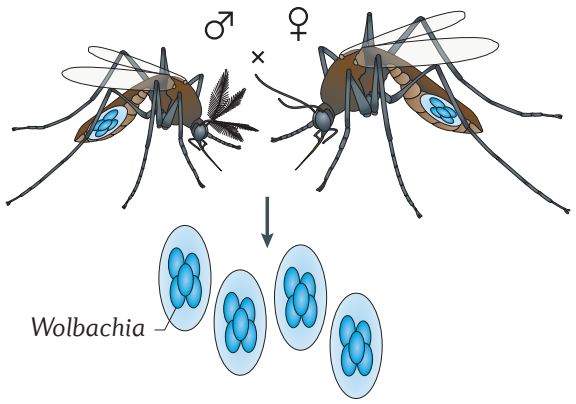

Wolbachia-infected offspring
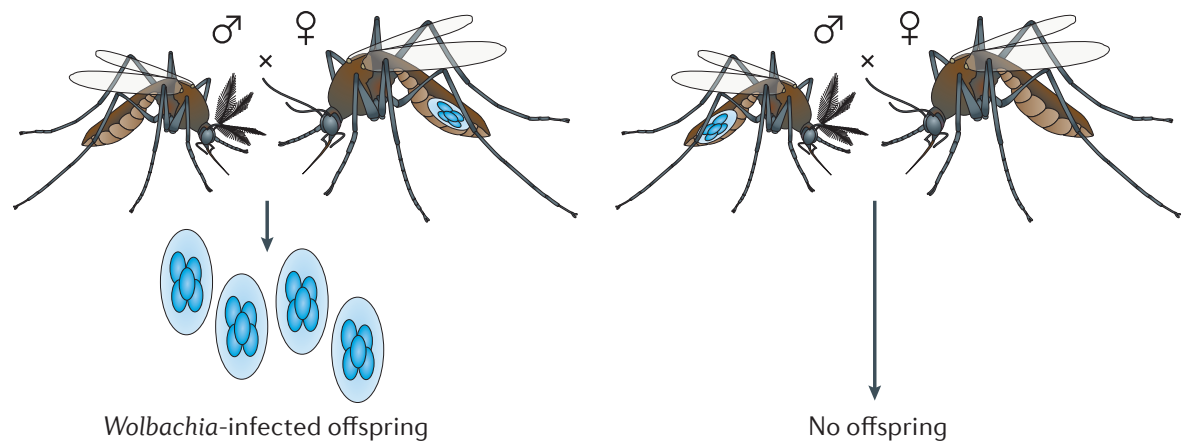

No offspring

Figure 6 | Wolbachia inheritance. Wolbachia are intracellular parasites that are inherited maternally. They spread through a population by killing offspring of Wolbachia-infected males unless they have mated with a female infected with the same strain of Wolbachia.

spread of a translocation-based gene drive system with a moderate fitness cost would be comparatively small (for example, just under $60 \%$ for a translocation homozygote with a $20 \%$ fitness cost) ${ }^{1}$. Overall, given their probable restriction to local populations and their ability to be removed completely by the release of large numbers of wild-type organisms, the development of underdominant gene drives (alone or in combination ${ }^{99,100}$ with other types of gene drives), and particularly of translocation drives, should be of high priority.

\section{Heritable microorganisms: Wolbachia}

In addition to the gene drives described above, all of which are integrated into the host nuclear genome, there are those that are cytoplasmically inherited. The best examples are the maternally inherited (FIG. 6), cytoplasmic, obligate intracellular bacteria parasites known as Wolbachia, which are predicted to reside in roughly half of all insects and can rapidly invade populations ${ }^{101}$. Originally discovered in mosquito populations in 1924 (REF. 102), Wolbachia have the ability to selfishly promote their existence in subsequent generations by manipulating and interfering with host reproduction capabilities. For example, certain strains of Wolbachia have been shown to induce male-killing, feminization, parthenogenesis and cytoplasmic incompatibility (FIG. 6) in various insect hosts9. In some cases, Wolbachia infections have also resulted in speciation ${ }^{13}$, reduced vector competence ${ }^{103-108}$ and even shortening of host lifespan ${ }^{109,110}$. These remarkable abilities are currently being exploited

Parthenogenesis

A type of asexual reproduction in which the offspring develop from an unfertilized egg.

Cytoplasmic incompatibility A phenomenon that results when sperm and eggs are unable to form viable offspring.

Transposons

A class of genetic elements

that can insert themselves into different locations in a genome.

B-chromosomes

Small extra chromosomes to the standard complement that occur in many organisms. for mosquito control. For example, A. aegypti purposely infected with the wMel strain of Wolbachia, which is known to significantly inhibit malaria and dengue transmission in the laboratory ${ }^{103}$, have been used to successfully invade wild A. aegypti populations in Australia, despite the mild fitness cost associated with the infection $^{111,112}$. Although using Wolbachia to control populations is a promising strategy, we are currently unable to genetically engineer these intracellular parasites. However, the flexibility of RNA-guided endonucleases may change this, potentially enabling the development of improved strains of Wolbachia with enhanced diseaserefractory properties and a reduced fitness impact on their host, allowing them to propagate more rapidly throughout an insect population.

\section{Other gene drive systems}

Several other promising gene drive systems have thus far only been advanced at the theoretical level. One of the most prominent is the use of transposons linked to a genetic payload, which would increase the frequency of the transposable element and genetic payload in the genome of a target organism, and eventually in the population ${ }^{113}$. However, transposable elements often have transposition rates that are too low to be usable ${ }^{10}$, are unpredictable owing to lack of control over their integration sites, and have proven to be difficult to mobilize after integration ${ }^{114}$. Supernumerary B-chromosomes have also been suggested as vehicles to carry payload genes ${ }^{13}$, as these small chromosomes are inherited at rates that are greater than Mendelian rates and can express transcripts ${ }^{15,116}$. However, they are poorly understood, making their engineering difficult. Various other hypothetical threshold-dependent gene drive systems have also been proposed. The Killer-Rescue system uses a toxin and an antidote gene at separate loci ${ }^{117}$. The inverse Medea system relies on a toxin that takes effect in the zygote unless it receives a maternally delivered antidote $^{118}$. The Merea system functions similarly to Medea, but the antidote to the maternal toxin is recessive ${ }^{119}$. The Semele system, conversely, uses a paternal semen-based toxin and a maternally delivered antidote ${ }^{119,120}$. Marshall and Hay ${ }^{121,122}$ have also proposed several additional variants utilizing toxin and antidote combinations, including the Medusa system, which induces a population crash by utilizing a pair of sex-linked toxins and antidotes ${ }^{122}$. In the future, RNA-guided nucleases may contribute to the development of each of these systems in diverse species.

\section{Safety and regulation}

Although gene drives demonstrate great promise as potential innovative solutions to difficult biological problems (BOX 1), they may also pose dangers. Payload genes may have unanticipated effects, and some gene drives have the potential to cause extinctions $s^{41,123,124}$. A gene drive may also have off-target effects, spread outside an expected geographical area, or traverse into another species ${ }^{49}$. Concerns have even been raised over the potential misuse of gene drives for the purposes of causing economic damage or even bioterrorism ${ }^{125}$. Whereas some have called for a moratorium on the research of gene drives or a restriction 
in the availability of technical information ${ }^{125}$, others have raised the point that further research in gene drive systems is needed to develop defences ${ }^{126}$.

Currently, the Cartagena Protocol contains regulations to be enacted by signatory countries for the control of genetically modified organisms, such as a prohibition on the release of organisms that can cross international boundaries ${ }^{41,127}$. However, as several types of gene drive systems are likely to spread invasively, and as a number of countries (such as the United States) are not signatories to the Cartagena Protocol, a new legal framework may be required. The US National Academy of Sciences has recently convened a panel to discuss the potential hazards and regulation of gene drives, and to make recommendations regarding their safe use. Although there is no legislation specifically referring to gene drives ${ }^{41}$, their usage requires the need for local consent ${ }^{123,128}$, and full transparency and early engagement with the public will be crucial for their approval. Transgenic insects have already been used successfully in the field for the sterile insect technique ${ }^{12,73,129-133}$, which may pave the way socially and legally for the release of organisms carrying gene drive systems. Although some communities have reacted positively to the use of genetically modified insects for temporary population suppression ${ }^{129,131,132}$, scepticism remains concerning the release of genetically modified organisms that may persist in the wild. One study indicated that concerns include enhanced disease severity and high costs, but that greater education and the promise of testing and field trials increased acceptance of the possible use of gene drives ${ }^{134}$.

To prevent escape of organisms carrying gene drive systems and to mitigate their accidental release, several safety precautions can be initiated ${ }^{40,135-137}$, including molecular confinement in laboratory organisms that are not designated for release into the wild; this approach has been pioneered in yeast ${ }^{16}$. The risk of unanticipated effects can be reduced, albeit not entirely prevented, by first conducting large-cage trials followed by initial field trials in isolated populations, such as those found on islands. Additionally, threshold-dependent drives such as underdominance systems, which would probably be confined to smaller breeding populations and can be removed fairly easily by releasing a large number of wild-type organisms, would be ideal for initial testing of engineered gene drives in the wild ${ }^{138,139}$ and in contexts where approval of transgenic organisms is limited ${ }^{123}$. Furthermore, before releasing gene drives that will spread invasively, it may be desirable to design second-generation reversability systems ${ }^{41,127}$. For example, a reversal gene drive system utilizing an RNA-guided endonuclease has already been created in yeast ${ }^{16}$.

\section{Conclusions}

Despite the potential benefits of gene drives in various crucial contexts (BOX 1), these systems remain understudied, and it remains unclear which type of system is a more appropriate candidate for development given the specific engineering limitations and particular ecological and regulatory situations.

Homing-based modification drives are efficient at spreading through populations, but they are also the least stable compared to other gene drive systems and the most likely to induce resistance alleles. Meiotic drive systems and homing-based suppression drives can both suppress or eliminate populations, which could bring about unanticipated ecological ramifications, and resistance alleles would also strongly be selected for. Medea is more stable and controllable than homing based drives, as it does not rely on DNA repair, but efforts to bring this system to medically or ecologically relevant organisms have met with difficulties. Underdominance systems can potentially be more easily confined to local breeding populations, are completely removable via the release of large numbers of wild-type organisms, and are highly evolutionarily stable in the form of translocation-based drives; however, they require large release thresholds, and application of these systems to species other than D. melanogaster may also be challenging.

RNA-guided endonucleases may provide new tools for the effective development of these various types of gene drive systems with characteristics that are amenable to the changing regulatory environment. Overall, success in this critically important field could greatly reduce the worldwide burden of infectious diseases and could provide other important economic and ecological benefits.
1. Curtis, C. F. Possible use of translocations to fix desirable genes in insect pest populations. Nature 218, 368-369 (1968).

2. Hamilton, W. D. Extraordinary sex ratios. A sex-ratio theory for sex linkage and inbreeding has new implications in cytogenetics and entomology. Science 156, 477-488 (1967).

3. Serebrovskii, A. S. On the possibility of a new method for the control of insect pests. Zool. Zhurnal 19, 618-630 (1940)

4. Burt, A. Site-specific selfish genes as tools for the control and genetic engineering of natural populations. Proc. Biol. Sci. 270, 921-928 (2003).

5. Huang, Y., Magori, K., Lloyd, A. L. \& Gould, F Introducing desirable transgenes into insect populations using Y-linked meiotic drive a theoretical assessment. Evolution 61, 717-726 (2007).

6. Caraballo, H. \& King, K. Emergency department management of mosquito-borne illness: malaria, dengue, and West Nile virus. Emerg. Med. Pract. 16 $1-23$ (2014).
7. Sinha, S., Medhi, B. \& Sehgal, R. Challenges of drugresistant malaria. Parasite 21, 61 (2014)

8. World Health Organization. Malaria. World Health Organization [online], http://www.who.int/mediacentre/ factsheets/fs094/en/ (2015)

9. Burt, A. Heritable strategies for controlling insect vectors of disease. Philos. Trans. R. Soc. Lond. B. Biol. Sci. 369, 20130432 (2014).

A good, concise review of gene drive systems.

10. Sinkins, S. P. \& Gould, F. Gene drive systems for insect disease vectors. Nat. Rev. Genet. 7, 427-435 (2006).

11. Alphey, L. Genetic control of mosquitoes. Annu. Rev. Entomol. 59, 205-224 (2014). A good review of gene drive systems and the sterile insect technique.

12. Alphey, L. et al. Genetic control of Aedes mosquitoes. Pathog. Glob. Health 107, 170-179 (2013).

13. Werren, J. H. Selfish genetic elements, genetic conflict, and evolutionary innovation. Proc. Natl Acad. Sci. USA 108 (Suppl. 2), 10863-10870 (2011).

14. Hurst, G. D. \& Werren, J. H. The role of selfish genetic elements in eukaryotic evolution. Nat. Rev. Genet. 2 , 597-606 (2001).
15. Marshall, J. M. \& Akbari, O. S. in Genetic Control of Malaria and Dengue (ed. Adelman, Z. N.) 169-196 (Academic Press, 2015).

16. DiCarlo, J. E., Chavez, A., Dietz, S. L., Esvelt, K. M. \& Church, G. M. Safeguarding CRISPR-Cas9 gene drives in yeast. Nat. Biotechnol. 33 1250-1255 (2015). The first creation of a homing drive in a microorganism.

17. Gantz, V. M. \& Bier, E. Genome editing. The mutagenic chain reaction: a method for converting heterozygous to homozygous mutations. Science $348,442-444$ (2015).

The first use of RNA-guided endonucleases in the creation of a homing drive.

18. Akbari, O. S. et al. A synthetic gene drive system for local, reversible modification and suppression of insect populations. Curr. Biol. 23, 671-677 (2013). The first engineered underdominance system created with a toxin-antitoxin mechanism.

19. Chen, C. H. et al. A synthetic maternal-effect selfish genetic element drives population replacement in Drosophila. Science 316, 597-600 (2007). The first engineered Medea gene drive system. 
20. Akbari, O. S. et al. Novel synthetic Medea selfish genetic elements drive population replacement in Drosophila; a theoretical exploration of Medea dependent population suppression. ACS Synth. Biol. 3, 915-928 (2014).

A study describing the creation of Medea systems with two novel developmental gene targets.

21. Gantz, V. M. et al. Highly efficient Cas9-mediated gen drive for population modification of the malaria vector mosquito Anopheles stephensi. Proc. Natl Acad. SCi. USA 112, E6736-E6743 (2015)

The first development of a modification type homing drive in a major disease vector.

22. Hammond, A. et al. A CRISPR-Cas9 gene drive system targeting female reproduction in the malaria mosquito vector Anopheles gambiae. Nat. Biotechnol. 34, 78-83 (2016) The first development of a suppression type homing drive in a major disease vector.

23. Mali, P., Esvelt, K. M. \& Church, G. M. Cas9 as a versatile tool for engineering biology. Nat. Methods 10, 957-963 (2013).

24. Li, J. F. et al. Multiplex and homologous recombination-mediated genome editing in Arabidopsis and Nicotiana benthamiana using guide RNA and Cas9. Nat. Biotechnol. 31, 688-691 (2013).

25. Frokjaer-Jensen, C. Exciting prospects for precise engineering of Caenorhabditis elegans genomes with CRISPR/Cas9. Genetics 195, 635-642 (2013).

26. Gratz, S. J. et al. Genome engineering of Drosophila with the CRISPR RNA-guided Cas9 nuclease. Genetics 194, 1029-1035 (2013)

27. Basu, S. et al. Silencing of end-joining repair for efficient site-specific gene insertion after TALEN/ CRISPR mutagenesis in Aedes aegypti. Proc. Nat Acad. Sci. USA 112, 4038-4043 (2015).

28. Kistler, K. E., Vosshall, L. B. \& Matthews, B. J. Genome engineering with CRISPR-Cas9 in the mosquito Aedes aegypti. Cell Rep. 11, 51-60 (2015).

29. Hwang, W. Y. et al. Efficient genome editing in zebrafish using a CRISPR-Cas system. Nat. Biotechnol. 31 227-229 (2013).

30. Shen, B. et al. Generation of gene-modified mice via Cas9/RNA-mediated gene targeting. Cell Res. 23 720-723 (2013).

31. Niu, Y. et al. Generation of gene-modified cynomolgus monkey via Cas9/RNA-mediated gene targeting in one-cell embryos. Cell 156, 836-843 (2014).

32. Cong, L. et al. Multiplex genome engineering using CRISPR/Cas systems. Science 339, 819-823 (2013).

33. O'Connell, M. R. et al. Programmable RNA recognition and cleavage by CRISPR/Cas9. Nature 516, 263-266 (2014).

34. Price, A. A., Sampson, T. R., Ratner, H. K., Grakoui, A \& Weiss, D. S. Cas9-mediated targeting of viral RNA in eukaryotic cells. Proc. Natl Acad. Sci. USA 112 6164-6169 (2015)

35. Gao, Y. \& Zhao, Y. Self-processing of ribozyme-flanked RNAs into guide RNAs in vitro and in vivo for CRISPRmediated genome editing. J. Integr. Plant Biol. 56, 343-349 (2014)

36. Xie, K., Minkenberg, B. \& Yang, Y. Boosting CRISPR/ Cas9 multiplex editing capability with the endogenous tRNA-processing system. Proc. Natl Acad. Sci. USA 112, 3570-3575 (2015)

37. Xie, S. Shen, B., Zhang, C., Huang, X. \& Zhang, Y. sgRNAcas9: a software package for designing CRISPR sgRNA and evaluating potential off-target cleavage sites. PLOS ONE 9 e 100448 (2014).

38. Zetsche, B. et al. Cpf1 is a single RNA-guided endonuclease of a class 2 CRISPR-Cas System. Cell 163, 759-771 (2015).

The discovery of a new type of RNA-guided endonuclease.

39. Bull, J. J. Evolutionary decay and the prospects for long-term disease intervention using engineered insect vectors. Evol. Med. Publ. Health 2015, 152-166 (2015).

40. Akbari, O. S. et al. Safeguarding gene drive experiments in the laboratory. Science 349, 927-929 (2015).

A comprehensive guide covering the safe creation of gene drives.

41. Oye, K. A. et al. Biotechnology. Regulating gene drives. Science 345, 626-628 (2014). A good discussion of the regulatory and politica environment for gene drive systems.

42. Stoddard, B. L. Homing endonucleases: from microbial genetic invaders to reagents for targeted DNA modification. Structure 19, 7-15 (2011).
43. Deredec, A., Godfray, H. C. \& Burt, A. Requirements for effective malaria control with homing endonuclease genes. Proc. Natl Acad. Sci. USA 108, E874-E880 (2011).

44. Deredec, A., Burt, A. \& Godfray, H. C. The population genetics of using homing endonuclease genes in vector and pest management. Genetics 179, 2013-2026 (2008)

45. North, A., Burt, A., Godfray, H. C. \& Buckley, Y. Modelling the spatial spread of a homing endonuclease gene in a mosquito population. J. Appl. Ecol. 50 1216-1225 (2013)

46. Unckless, R. L., Messer, P. W., Connallon, T. \& Clark, A. G. Modeling the manipulation of natural populations by the mutagenic chain reaction. Genetics 201, 425-431 (2015)

47. Alphey, N. \& Bonsall, M. B. Interplay of population genetics and dynamics in the genetic control of mosquitoes. J. R. Soc. Interface 11, 20131071 (2014).

48. Gantz, V. M. \& Bier, E. The dawn of active genetics. Bioessays 38, 50-63 (2016).

49. Esvelt, K. M., Smidler, A. L., Catteruccia, F. \& Church, G. M. Concerning RNA-guided gene drives for the alteration of wild populations. eLife 3, e03401 (2014).

A good review covering the use of RNA-guided endonucleases in gene drive systems.

50. Windbichler, N. et al. A synthetic homing endonuclease-based gene drive system in the human malaria mosquito. Nature 473, 212-215 (2011).

51. Windbichler, N. et al. Homing endonuclease mediated gene targeting in Anopheles gambiae cells and embryos. Nucleic Acids Res. 35, 5922-5933 (2007)

52. Chan, Y. S., Huen, D. S., Glauert, R., Whiteway, E. \& Russell, S. Optimising homing endonuclease gene drive performance in a semi-refractory species: the Drosophila melanogaster experience. PLOS ONE 8 e54130 (2013)

53. Chan, Y. S., Naujoks, D. A., Huen, D. S. \& Russell, S Insect population control by homing endonucleasebased gene drive: an evaluation in Drosophila melanogaster. Genetics 188, 33-44 (2011).

54. Chan, Y. S. et al. The design and in vivo evaluation of engineered I-Onul-based enzymes for HEG gene drive. PLOS ONE 8, e74254 (2013).

55. Simoni, A. et al. Development of synthetic selfish elements based on modular nucleases in Drosophila melanogaster. Nucleic Acids Res. 42, 7461-7472 (2014)

56. Jinek, M. et al. A programmable dual-RNA-guided DNA endonuclease in adaptive bacterial immunity. Science 337, 816-821 (2012).

57. Bae, S., Kweon, J., Kim, H. S. \& Kim, J. S. Microhomology-based choice of Cas9 nuclease target sites. Nat. Methods 11, 705-706 (2014).

58. Akbari, O. S., Papathanos, P. A., Sandler, J. E. Kennedy, K. ¿ Hay, B. A. Identification of germline transcriptional regulatory elements in Aedes aegypt Sci. Rep. 4, 3954 (2014).

59. Biedler, J. K., Oi, Y., Pledger, D., James, A. A. \& Tu, Z Maternal germline-specific genes in the Asian malaria mosquito Anopheles stephensi: characterization and application for disease control. G3 (Bethesda) 5 157-166 (2015)

60. Chu, V. T. et al. Increasing the efficiency of homologydirected repair for CRISPR-Cas9-induced precise gene editing in mammalian cells. Nat. Biotechnol. 33 543-548 (2015).

61. Bae, S., Park, J. \& Kim, J. S. Cas-OFFinder: a fast and versatile algorithm that searches for potential off-target sites of Cas9 RNA-guided endonucleases. Bioinformatics 30, 1473-1475 (2014).

62. Tsai, S. Q. et al. GUIDE-seq enables genome-wide profiling of off-target cleavage by CRISPR-Cas nucleases. Nat. Biotechnol. 33, 187-197 (2015).

63. Davis, K. M., Pattanayak, V., Thompson, D. B., Zuris, J. A. \& Liu, D. R. Small molecule-triggered Cas9 protein with improved genome-editing specificity. Nat. Chem. Biol. 11, 316-318 (2015)

64. Slaymaker, I. M. et al. Rationally engineered Cas 9 nucleases with improved specificity. Science $\mathbf{3 5 1}$, 84-88 (2015)

65. Lyttle, T. W. Cheaters sometimes prosper: distortion of mendelian segregation by meiotic drive. Trends Genet. 9. 205-210 (1993).

66. Papathanos, P. A., Windbichler, N. \& Akbari, O. S. in Transgenic Insects: Techniques and Applications (ed. Benedict, M. Q.) 83-100 (CABI, 2014).

67. Wood, R. J. \& Newton, M. E. Sex-ratio distortion caused by meiotic drive in mosquitoes. Am. Naturalist 137, 379-391 (1991).
68. Helleu, Q., Gerard, P. R. \& Montchamp-Moreau, C. Sex chromosome drive. Cold Spring Harb. Perspect. Biol. 7, a017616 (2015)

69. Craig, G. B. Jr., Hickey, W. A. \& Vandehey, R. C. An inherited male-producing factor in Aedes aegypti. Science 132, 1887-1889 (1960).

70. Hickey, W. A. \& Craig, G. B. Jr. Distortion of sex ratio in populations of Aedes aegypti. Can. J. Genet. Cytol. 8, 260-278 (1966).

71. Lyttle, T. W. Experimental population genetics of meiotic drive systems. I. Pseudo-Y chromosomal drive as a means of eliminating cage populations of Drosophila melanogaster. Genetics 86, 413-445 (1977).

72. Newton, M. E., Wood, R. J. \& Southern, D. I. A cytogenetic analysis of meiotic drive in the mosquito Aedes aegypti (L.). Genetica 46, 297-318 (1976).

73. Alphey, L., Nimmo, D., O'Connell, S. \& Alphey, N. Insect population suppression using engineered insects. Adv. Exp. Med. Biol. 627, 93-103 (2008)

74. Klein, T. A., Windbichler, N., Deredec, A., Burt, A. \& Benedict, M. Q. Infertility resulting from transgenic I-Ppol male Anopheles gambiae in large cage trials. Pathog. Glob. Health 106, 20-31 (2012).

75. Windbichler, N., Papathanos, P. A. \& Crisanti, A. Targeting the $\mathrm{X}$ chromosome during spermatogenesis induces $\mathrm{Y}$ chromosome transmission ratio distortion and early dominant embryo lethality in Anopheles gambiae. PLoS Genet. 4, e1000291 (2008).

76. Galizi, R. et al. A synthetic sex ratio distortion system for the control of the human malaria mosquito. Nat. Commun. 5, 3977 (2014). The creation of a fully functional autosomallocalized X-shredder in mosquitoes.

77. Bernardini, F. et al. Site-specific genetic engineering of the Anopheles gambiae Y chromosome. Proc. Natl Acad. Sci. USA 111, 7600-7605 (2014)

78. Wade, M. J. \& Beeman, R. W. The population dynamics of maternal-effect selfish genes. Genetics 138, 1309-1314 (1994).

79. Hay, B. A. et al. Engineering the genomes of wild insect populations: challenges, and opportunities provided by synthetic Medea selfish genetic elements. J. Insect Physiol. 56, 1402-1413 (2010).

80. Ward, C. M. et al. Medea selfish genetic elements as tools for altering traits of wild populations: a theoretical analysis. Evolution 65, 1149-1162 (2011)

81. Guevara-Souza, M. \& Vallejo, E. E. Computer simulation on disease vector population replacement driven by the maternal effect dominant embryonic arrest. Adv. Exp. Med. Biol. 696, 335-343 (2011).

82. Huang, Y., Lloyd, A. L., Legros, M. \& Gould, F. Genedrive in age-structured insect populations. Evol. Appl. 2, 143-159 (2009)

83. Legros, M. et al. Modeling the dynamics of a nonlimited and a self-limited gene drive system in structured Aedes aegypti populations. PLOS ONE 8 e83354 (2013).

84. Altrock, P. M., Traulsen, A. \& Reed, F. A. Stability properties of underdominance in finite subdivided populations. PLoS Comput. Biol. 7, e1002260 (2011)

85. Altrock, P. M., Traulsen, A., Reeves, R. G. \& Reed, F. A. Using underdominance to bi-stably transform local populations. J. Theor. Biol. 267 62-75 (2010)

86. Magori, K. \& Gould, F. Genetically engineered underdominance for manipulation of pest populations: a deterministic model. Genetics 172, 2613-2620 (2006).

87. Davis, S., Bax, N. \& Grewe, P. Engineered underdominance allows efficient and economical introgression of traits into pest populations. J. Theor. Biol. 212, 83-98 (2001).

88. Huang, Y., Lloyd, A. L., Legros, M. \& Gould, F. Genedrive into insect populations with age and spatial structure: a theoretical assessment. Evol. Appl. 4 415-428 (2011).

89. Foster, G. G., Whitten, M. J., Prout, T. \& Gill, R. Chromosome rearrangements for the control of insect pests. Science 176, 875-880 (1972).

90. Coghlan, A., Eichler, E. E., Oliver, S. G., Paterson, A. H. $\&$ Stein, L. Chromosome evolution in eukaryotes: a multi-kingdom perspective. Trends Genet. 21 673-682 (2005)

91. Gould, F. \& Schliekelman, P. Population genetics of autocidal control and strain replacement. Annu. Rev. Entomol. 49, 193-217 (2004)

92. Asman, S. M., McDonald, P. T. \& Prout, T. Field studies of genetic control systems for mosquitoes. Annu. Rev. Entomol. 26, 289-318 (1981) 
93. Baker, R. H. Chromosome rearrangements in the control of mosquitoes. Prev. Vet. Med. 2, 529-540 (1984).

94. Laven, H., Cousserans, J. \& Guille, G. Eradicating mosquitoes using translocations: a first field experiment Nature 236, 456-457 (1972).

95. Robinson, A. S. Progress in the use of chromosomal translocations for the control of insect pests. Biol. Rev. Camb. Philos. Soc. 51, 1-24 (1976).

96. Reeves, R. G., Bryk, J., Altrock, P. M., Denton, J. A. \& Reed, F. A. First steps towards underdominant genetic transformation of insect populations. PLOS ONE 9, e97557 (2014).

97. Egli, D., Hafen, E. \& Schaffner, W. An efficient method to generate chromosomal rearrangements by targeted DNA double-strand breaks in Drosophila melanogaster. Genome Res. 14, 1382-1393 (2004).

98. Sherizen, D., Jang, J. K., Bhagat, R., Kato, N. \& McKim, K. S. Meiotic recombination in Drosophila females depends on chromosome continuity between genetically defined boundaries. Genetics 169 , 767-781 (2005)

99. Gokhale, C. S., Reeves, R. G. \& Reed, F. A. Dynamics of a combined Medea-underdominant population transformation system. BMC Evol. Biol. 14, 98 (2014).

100. Huang, Y., Magori, K., Lloyd, A. L. \& Gould, F. Introducing transgenes into insect populations using combined gene-drive strategies: modeling and analysis. Insect Biochem. Mol. Biol. 37, 1054-1063 (2007).

101. Serbus, L. R., Casper-Lindley, C., Landmann, F. $\&$ Sullivan, W. The genetics and cell biology of Wolbachia-host interactions. Annu. Rev. Genet. 42 , 683-707 (2008)

102. Hertig, M. \& Wolbach, S. B. Studies on Rickettsia-like micro-organisms in insects. J. Med. Res. 44, 329-374 (1924).

103. Moreira, L. A. et al. A Wolbachia symbiont in Aedes aegypti limits infection with dengue, Chikungunya, and Plasmodium. Cell 139, 1268-1278 (2009).

104. Bian, G. et al. Wolbachia invades Anopheles stephensi populations and induces refractoriness to Plasmodium infection. Science 340, 748-751 (2013).

105. Bian, G., Zhou, G., Lu, P. \& Xi, Z. Replacing a native Wolbachia with a novel strain results in an increase in endosymbiont load and resistance to dengue virus in a mosquito vector. PLoS Negl. Trop. Dis. 7, e2250 (2013)

106. Blagrove, M. S., Arias-Goeta, C., Failloux, A. B. $\&$ Sinkins, S. P. Wolbachia strain wMel induces cytoplasmic incompatibility and blocks dengue transmission in Aedes albopictus. Proc. Natl Acad. Sci. USA 109, 255-260 (2012).

107. Kambris, Z., Cook, P. E., Phuc, H. K. \& Sinkins, S. P. Immune activation by life-shortening Wolbachia and reduced filarial competence in mosquitoes. Science 326, 134-136 (2009).

108. Sinkins, S. P. Wolbachia and arbovirus inhibition in mosquitoes. Future Microbiol. 8, 1249-1256 (2013).

109. McMeniman, C. J. et al. Stable introduction of a life shortening Wolbachia infection into the mosquito Aedes aegypti. Science 323, 141-144 (2009).

110. Rasgon, J. L., Styer, L. M. \& Scott, T. W. Wolbachiainduced mortality as a mechanism to modulate pathogen transmission by vector arthropods. J. Med. Entomol. 40, 125-132 (2003).

111. Hoffmann, A. A. et al. Successful establishment of Wolbachia in Aedes populations to suppress dengue transmission. Nature 476, 454-457 (2011).
112. Walker, T. et al. The wMel Wolbachia strain blocks dengue and invades caged Aedes aegypti populations. Nature 476, 450-453 (2011).

113. Rasgon, J. L. \& Gould, F. Transposable element insertion location bias and the dynamics of gene drive in mosquito populations. Insect Mol. Biol. 14 , 493-500 (2005).

114. Smith, R. C. \& Atkinson, P. W. Mobility properties of the Hermes transposable element in transgenic lines of Aedes aegypti. Genetica 139, 7-22 (2011).

115. Houben, A., Banaei-Moghaddam, A. M., Klemme, S. \& Timmis, J. N. Evolution and biology of supernumerary B chromosomes. Cell. Mol. Life Sci. 71, 467-478 (2014).

116. Akbari, O. S., Antoshechkin, I., Hay, B. A. \& Ferree, P. M. Transcriptome profiling of Nasonia vitripennis testis reveals novel transcripts expressed from the selfish $B$ chromosome, paternal sex ratio. G3 (Bethesda) 3, 1597-1605 (2013).

117. Gould, F., Huang, Y., Legros, M. \& Lloyd, A. L. A killerrescue system for self-limiting gene drive of antipathogen constructs. Proc. Biol. Sci. 275, 2823-2829 (2008)

118. Marshall, J. M. \& Hay, B. A. Inverse Medea as a novel gene drive system for local population replacement: a theoretical analysis. J. Hered 102, 336-341 (2011).

119. Marshall, J. M. The toxin and antidote puzzle: new ways to control insect pest populations through manipulating inheritance. Bioeng. Bugs 2, 235-240 (2011).

120. Marshall, J. M., Pittman, G. W., Buchman, A. B. \& Hay, B. A. Semele: a killer-male, rescue-female system for suppression and replacement of insect disease vector populations. Genetics 187, 535-551 (2011).

121. Marshall, J. M. \& Hay, B. A. General principles of single-construct chromosomal gene drive. Evolution 66, 2150-2166 (2012).

122. Marshall, J. M. \& Hay, B. A. Medusa: a novel gene drive system for confined suppression of insect populations. PLOS ONE 9, e102694 (2014).

123. Knols, B. G., Bossin, H. C., Mukabana, W. R. \& Robinson, A. S. Transgenic mosquitoes and the fight against malaria: managing technology push in a turbulent GMO world. Am. J. Trop. Med. Hyg. 77 232-242 (2007)

124. Webber, B. L., Raghu, S. \& Edwards, O. R. Opinion: is CRISPR-based gene drive a biocontrol silver bullet or global conservation threat? Proc. Natl Acad. Sci. USA 112, 10565-10567 (2015).

125. Gurwitz, D. Gene drives raise dual-use concerns Science 345, 1010 (2014).

126. Oye, K. A. \& Esvelt, K. M. Gene drives raise dual-use concerns-response. Science 345, 1010-1011 (2014).

127. Marshall, J. M. The Cartagena Protocol and genetically modified mosquitoes. Nat. Biotechnol. 28, 896-897 (2010).

128. McNaughton, D. The importance of long-term social research in enabling participation and developing engagement strategies for new dengue control technologies. PLoS Negl. Trop. Dis. 6, e1785 (2012).

129. Carvalho, D. O. et al. Suppression of a field population of Aedes aegypti in Brazil by sustained release of transgenic male mosquitoes. PLoS Negl. Trop. Dis. 9, e0003864 (2015)

130. Harris, A. F. et al. Successful suppression of a field mosquito population by sustained release of engineered male mosquitoes. Nat. Biotechnol. 30 828-830 (2012).

131. Harris, A. F. et al. Field performance of engineered male mosquitoes. Nat. Biotechnol. 29, 1034-1037 (2011).
132. Lacroix, R. et al. Open field release of genetically engineered sterile male Aedes aegypti in Malaysia. PLOS ONE 7, e42771 (2012)

133. Simmons, G. S. et al. Field performance of a genetically engineered strain of pink bollworm PLOS ONE 6, e24110 (2011)

134. Marshall, J. M., Toure, M. B., Traore, M. M., Famenini, S. \& Taylor, C. E. Perspectives of people in Mali toward genetically-modified mosquitoes for malaria control. Malar. J. 9, 128 (2010).

135. James, A. A. Gene drive systems in mosquitoes: rules of the road. Trends Parasitol. 21, 64-67 (2005)

136. David, A. S., Kaser, J. M., Morey, A. C., Roth, A. M $\Sigma$ Andow, D. A. Release of genetically engineered insects: a framework to identify potential ecological effects. Ecol. Evol. 3, 4000-4015 (2013).

137. Wolbers, M., Kleinschmidt, I., Simmons, C. P. \& Donnelly, C. A. Considerations in the design of clinical trials to test novel entomological approaches to dengue control. PLoS Negl. Trop. Dis. 6, e1937 (2012).

138. Marshall, J. M. The effect of gene drive on containment of transgenic mosquitoes. J. Theor. Biol. 258, 250-265 (2009).

139. Marshall, J. M. \& Hay, B. A. Confinement of gene drive systems to local populations: a comparative analysis. J. Theor. Biol. 294, 153-171 (2012).

140. Fuchs, S., Nolan, T. \& Crisanti, A. Mosquito transgenic technologies to reduce Plasmodium transmission. Methods Mol. Biol. 923, 601-622 (2013).

141. Wang, S. \& Jacobs-Lorena, M. Genetic approaches to interfere with malaria transmission by vector mosquitoes. Trends Biotechnol. 31, 185-193 (2013).

142. Corby-Harris, V. et al. Activation of Akt signaling reduces the prevalence and intensity of malaria parasite infection and lifespan in Anopheles stephensi mosquitoes. PLoS Pathog. 6, e1001003 (2010).

143. Sumitani, M. et al. Reduction of malaria transmission by transgenic mosquitoes expressing an antisporozoite antibody in their salivary glands. Insect Mol. Biol. 22, 41-51 (2013).

144. Franz, A. W., Balaraman, V. \& Fraser, M. J. Jr. Disruption of dengue virus transmission by mosquitoes. Curr. Opin. Insect Sci. 8, 88-96 (2015).

145. Franz, A. W. et al. Fitness impact and stability of a transgene conferring resistance to dengue- 2 virus following introgression into a genetically diverse Aedes aegypti strain. PLoS Negl. Trop. Dis. 8, e2833 (2014).

146. Grafton-Cardwell, E. E., Stelinski, L. L. \& Stansly, P. A Biology and management of Asian citrus psyllid, vector of the huanglongbing pathogens. Annu. Rev. Entomol. 58, 413-432 (2013).

147. Rosenblum, E. B. et al. Complex history of the amphibian-killing chytrid fungus revealed with genome resequencing data. Proc. Natl Acad. Sci. USA 110, 9385-9390 (2013).

148. Guirouilh-Barbat, J., Lambert, S., Bertrand, P. \& Lopez, B. S. Is homologous recombination really an error-free process? Front. Genet. 5, 175 (2014).

\section{Acknowledgements}

This work was supported by generous University of California, Riverside (UCR) laboratory start-up funds, a private donation by MaxMind, and a US National Institutes of Health (NIH) K22 grant (5K22AI113060-02) awarded to O.S.A. The authors are appreciative of the editor and four anonymous reviewers for their extensive comments, which greatly improved this Review.

Competing interests statement

The authors declare no competing interests. 